\title{
Prediction of keratoconus progression using deep learning of anterior segment optical coherence tomography maps
}

\author{
Kazutaka Kamiya ${ }^{1}$, Yuji Ayatsuka ${ }^{2}$, Yudai Kato ${ }^{2}$, Nobuyuki Shoji ${ }^{3}$, Takashi Miyai ${ }^{4}$, Hitoha Ishii ${ }^{4}$, \\ Yosai Mori ${ }^{5}$, Kazunori Miyata ${ }^{5}$ \\ ${ }^{1}$ Visual Physiology, Kitasato University, School of Allied Health Sciences, Kanagawa, Japan; ${ }^{2}$ Cresco Ltd., Tokyo, Japan; ${ }^{3}$ Department of \\ Ophthalmology, Kitasato University, School of Medicine, Kanagawa, Japan; ${ }^{4}$ Department of Ophthalmology, Tokyo University, School of Medicine, \\ Tokyo, Japan; ${ }^{5}$ Department of Ophthalmology, Miyata Eye Hospital, Miyazaki, Japan \\ Contributions: (I) Conception and design: K Kamiya, K Miyata; (II) Administrative support: N Shoji, K Miyata; (III) Provision of study materials or \\ patients: T Miyai, H Ishii, Y Mori, K Miyata; (IV) Collection and assembly of data: T Miyai, H Ishii, Y Mori; (V) Data analysis and interpretation: Y \\ Ayatsuka, Y Kato; (VI) Manuscript writing: All authors; (VII) Final approval of manuscript: All authors. \\ Correspondence to: Kazutaka Kamiya, MD, PhD. Professor, Visual Physiology, School of Allied Health Sciences, Kitasato University, 1-15-1 Kitasato, \\ Minami, Sagamihara, Kanagawa 252-0373, Japan. Email: kamiyak-tky@umin.ac.jp.
}

\begin{abstract}
Background: To predict keratoconus progression using deep learning of the color-coded maps measured with a swept-source anterior segment optical coherence tomography (As-OCT) device.

Methods: We enrolled 218 keratoconic eyes with and without disease progression. Using deep learning of the 6 color-coded maps (anterior elevation, anterior curvature, posterior elevation, posterior curvature, total refractive power, and pachymetry map) obtained by the As-OCT (CASIA, Tomey), we assessed the accuracy, sensitivity, and specificity of prediction of keratoconus progression in such eyes.

Results: Deep learning of the 6 color-coded maps exhibited an accuracy of 0.794 in discriminating keratoconus with and without progression. For a single map analysis, posterior elevation map (0.798) showed the highest accuracy, followed by anterior curvature map (0.775), posterior corneal curvature map (0.757), anterior elevation map (0.752), total refractive power map (0.729), and pachymetry map (0.720), in distinguishing between progressive and non-progressive keratoconus. The use of the adjusted algorithm by age subgroups improved to an accuracy of 0.849 .

Conclusions: Deep learning of the As-OCT color-coded maps effectively discriminates progressive keratoconus from non-progressive keratoconus with an accuracy of approximately $85 \%$ using the adjusted age algorithm, indicating that it will become an aid for predicting the progression of the disease, which is clinically beneficial for decision-making of the surgical indication of corneal cross-linking (CXL).
\end{abstract}

Keywords: Deep learning; keratoconus; progression; prediction; optical coherence tomography

Submitted Apr 11, 2021. Accepted for publication Jun 11, 2021.

doi: 10.21037/atm-21-1772

View this article at: https://dx.doi.org/10.21037/atm-21-1772

\section{Introduction}

Keratoconus is an ophthalmic disease commonly encountered in a daily clinical setting (1-3). This disease is mostly characterized by anterior bulging and progressive thinning of the cornea, and causes severe visual deterioration due to high myopic and irregular astigmatism, especially when the stage of the disease has become advanced over time.

Corneal cross-linking (CXL) has been developed to halt the progression of keratoconus and iatrogenic keratectasia (4). It has become a standard of care for such patients all over the world. Understanding the disease progression is mandatory to precisely determine the surgical indication of the CXL treatment. However, it is impractical, even for corneal specialists, to accurately differentiate progressive 
keratoconus from non-progressive keratoconus, based on the corneal measurements during a single visit. Accordingly, it is still necessary to carefully monitor the time-course of changes in the keratometric readings, corneal thickness, and manifest refraction, in an individual patient, to verify the indication for the CXL treatment. However, this longitudinal follow-up takes much time and is costly and inefficient, placing a burden on both the patients and the ophthalmologists involved.

Deep learning is one of the machine learning methodologies to use the training of multilayered neural networks. Especially, deep convolutional neural networks have been successfully employed for image recognition to predict a targeted outcome, in a variety of scientific fields (5). Anterior segment optical coherence tomography (As-OCT) is currently considered to be one of the best technologies that can precisely measure the curvature and the elevation of the anterior and posterior corneal surfaces, as well as the corneal thickness, even in eyes with opaque cornea $(6,7)$. We recently showed that deep learning of As-OCT images was highly effective not only as a screening test but also for determining the stage of keratoconus (8). Building upon this work, we postulate that the combined use of deep learning and As-OCT images during a single visit may also be clinically beneficial for detecting keratoconus progression. If proven, this may lead to the development of an important decision-making tool to determine the use of CXL treatment amongst keratoconic patients, since we currently have no other effective means for estimating keratoconus progression. The goal of this pilot study is to evaluate the prediction accuracy of deep learning of the As-OCT color-coded maps, to distinguish progressive keratoconus from nonprogressive keratoconus. We present the following article in accordance with the STARD reporting checklist (available at https://dx.doi.org/10.21037/atm-21-1772).

\section{Methods}

\section{Study population}

We registered the study protocol with the University Hospital Medical Information Network Clinical Trial Registry (000040308). We enrolled a total of two hundred eighteen eyes of 218 consecutive keratoconic patients, who had routine follow-up examinations for at least 1-year at Miyata Eye Hospital and Tokyo University Hospital, with good quality scans of corneal color-coded maps (anterior elevation, anterior curvature, posterior elevation, posterior curvature, total refractive power, and pachymetry maps) obtained by a swept-source As-OCT (CASIA SS- $1000^{\mathrm{TM}}$, Tomey, Aichi, Japan). Multiple corneal specialists diagnosed keratoconus with distinctive features (e.g., corneal colorcoded map with asymmetric bow-tie pattern with or without skewed axes), and at least one keratoconus sign (e.g., stromal thinning, conical bulging, Fleischer ring, Vogt striae, or apical scar) (9). Progression of keratoconus was defined as a $\geq 1.00$ diopter (D) increase in the maximum anterior curvature on corneal tomography or worsened corrected visual acuity accompanied by a $\geq 1.00 \mathrm{D}$ increase in astigmatism confirmed with 2 or more examinations over the previous 12 months (8). We divided the patients into two groups [progressive (156 eyes) and non-progressive (62 eyes) keratoconus groups], based on the above criteria. We requested these patients to stop wearing rigid contact lenses at least for 3 weeks before this measurement, to exclude the effect of such contact lenses as much as possible. This retrospective data review was approved by the Institutional Review Board of Miyata Eye Hospital (CS-315) and that of Tokyo University Hospital (2019354NI) and followed the tenets of the Declaration of Helsinki (as revised in 2013). Informed consent was obtained by the opt-out method.

\section{Anterior segment optical coherence tomography imaging}

We acquired 6 color-coded images anterior elevation map [ -130 to $130 \mu \mathrm{m}, 5 \mu \mathrm{m}$ step], anterior curvature map [9.0 to $101.5 \mathrm{D}, 5 \mathrm{D}$ step (35.5 to $50.5 \mathrm{D}, 1.5 \mathrm{D}$ step)], posterior elevation map [-260 to $260 \mu \mathrm{m}, 10 \mu \mathrm{m}$ step], posterior curvature map [ -3.0 to $-10.5 \mathrm{D}, 0.3 \mathrm{D}$ step], total refractive power map [9.0 to $101.5 \mathrm{D}, 5 \mathrm{D}$ step (35.5 to $50.5 \mathrm{D}, 1.5 \mathrm{D}$ step)], and pachymetry map [340 to $840 \mu \mathrm{m}$, $20 \mu \mathrm{m}$ step]\} (Figure 1), as described previously (8). In brief, this As-OCT uses a wavelength of $1,310 \mathrm{~nm}$ and has an axial resolution of $10 \mu \mathrm{m}$, a transverse resolution of $30 \mu \mathrm{m}$, and a scan rate of 30,000 A-scans/sec. After the examiner confirmed good alignment, it automatically started the measurements and processed the acquired data to convert the cross-sectional images. We selected one examination with a high image quality score according to the manufacturer's instructions.

\section{Classification of images by deep learning}

We obtained 218 As-OCT image sets (156 progressive samples and 62 non-progressive samples). These sets were 

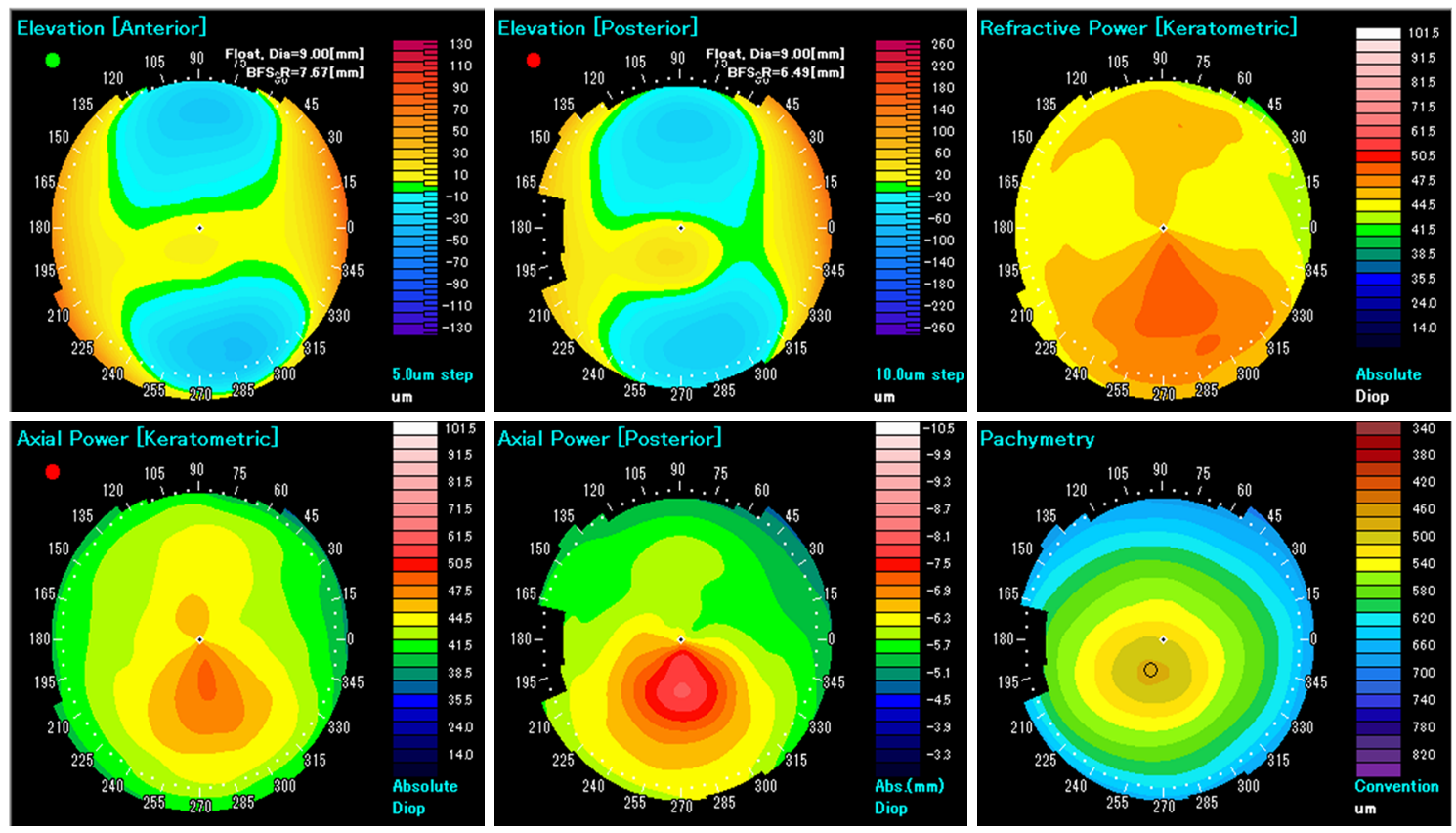

Figure 1 Representative example of 6 color-coded maps (anterior elevation, anterior curvature, posterior elevation, posterior curvature, total refractive power, and pachymetry map) obtained by an anterior segment optical coherence tomography. A color-scale bar was excluded in each map for deep learning.

split into 5 groups for $\mathrm{k}$-fold cross validation $(\mathrm{k}=5)$.

We used the Xception architecture for the neural network model (10). We trained machine learning models separately for each image type after eliminating a colorscaled bar, and we generated a total of 6 classifiers. These classifiers were trained to output 0.00 for non-progressive samples and 1.00 for progressive samples, and based on this they produced an output value in the range from 0.00 to 1.00 . Basically, a value of 0.50 or more was assessed as 'progressive'.

\section{Adjusted algoritbm by age subgroups}

Based on the histograms of the 6 classifiers' output for progressive and non-progressive samples according to 4 age ( $\leq 20$ years, 20 to 30 years, 30 to 40 years, and $>40$ years) subgroups, we additionally designed an adjusted algorithm that contains a kind of decision tree, using the classifiers' output and patient age, since it was possible to more precisely classify the As-OCT images when we select appropriate classifiers and thresholds according to age subgroups. In the first step of the tree, all input data were separated into each age subgroup. The rest of the tree was constructed based on the distributions of the histograms of the 6 classifiers' output (Figure 2).

\section{Statistical analysis}

Statistical analyses were performed using a statistical software (BellCurve for Excel, Social Survey Research Information Co., Ltd., Tokyo, Japan). The Welch's $t$-test was used to compare patient age, and the Fisher's exact test to compare the percentages of female, between the progressive and non-progressive groups. The results are expressed as mean \pm standard deviation, and a value of $\mathrm{P}<0.05$ was considered statistically significant.

\section{Results}

Table 1 shows the output data of deep learning in distinguishing between progressive and non-progressive keratoconus. Patient age was $25.2 \pm 9.9$ and $34.8 \pm 15.1$ years, in the progressive and non-progressive groups, respectively (Welch's $t$-test, $\mathrm{P}<0.001)$. The percentage of female was $24.4 \%$ and $37.1 \%$, in the progressive and non-progressive groups, respectively (Fisher's exact test, $\mathrm{P}=0.067$ ). Deep learning of these 6 color-coded maps exhibited an accuracy of 0.794 in discriminating between progressive and non- 


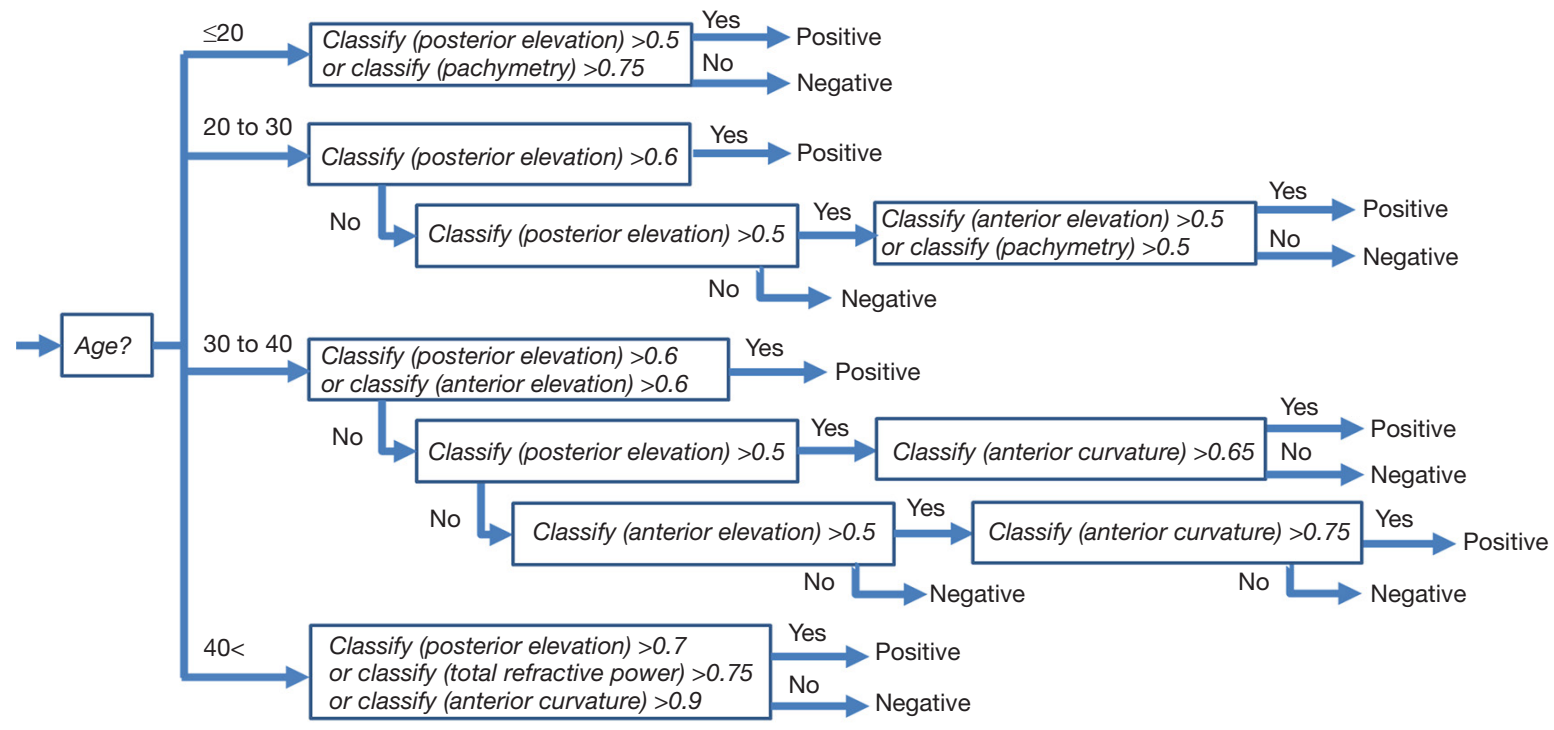

Figure 2 Adjusted algorithm using the classifiers' output according to age subgroups.

Table 1 The output data of deep learning in distinguishing between progressive and non-progressive keratoconus

\begin{tabular}{|c|c|c|c|c|c|c|}
\hline Actual category & Positive & Negative & Accuracy & Sensitivity & Specificity & Precision \\
\hline \multicolumn{7}{|c|}{ No adjusted algorithm } \\
\hline Progressive & 147 & 9 & 0.794 & 0.942 & 0.419 & 0.803 \\
\hline Non-progressive & 36 & 26 & & & & \\
\hline Progressive & 149 & 7 & 0.849 & 0.955 & 0.581 & 0.851 \\
\hline Non-progressive & 26 & 36 & & & & \\
\hline
\end{tabular}

progressive keratoconus if we defined progression when 5 or more of the 6 color-coded maps showed progression (Table 2). Posterior elevation map (0.798) provided the highest accuracy, followed by anterior curvature map (0.775), posterior corneal curvature map (0.757), anterior elevation map (0.752), total refractive power map (0.729), and pachymetry map (0.720), in discriminating between progressive and non-progressive keratoconus.

Figures 3-8 show the histograms of the 6 classifiers' output for progressive and non-progressive samples, according to age subgroups. When we applied this adjusted algorithm of the 6 classifiers, the accuracy was improved from 0.794 to 0.849 , as a result of much improvement of the specificity without reducing the sensitivity.

\section{Discussion}

In the current study, our findings demonstrated that deep learning of 6 color-coded maps obtained by the As-OCT was an effective method for discriminating the presence or the absence of progression of the disease and that posterior elevation map exhibited the highest accuracy to distinguish between progressive and non-progressive keratoconus, followed by anterior curvature map, posterior corneal curvature map, anterior elevation map, total refractive power map, and pachymetry map, for a single map analysis.

Deep learning, one of the machine learning techniques dealing with the training of multi-layer artificial neural networks, has also been employed in numerous 
Table 2 The accuracy, the sensitivity, the specificity, and the precision outcomes in predicting keratoconus progression by a single map analysis

\begin{tabular}{|c|c|c|c|c|c|c|}
\hline Actual category & Positive & Negative & Accuracy & Sensitivity & Specificity & Precision \\
\hline Progressive & 152 & 4 & 0.752 & 0.974 & 0.194 & 0.752 \\
\hline Non-progressive & 50 & 12 & & & & \\
\hline \multicolumn{7}{|l|}{ Anterior curvature } \\
\hline Non-progressive & 44 & 18 & & & & \\
\hline \multicolumn{7}{|l|}{ Posterior elevation } \\
\hline Progressive & 148 & 8 & 0.798 & 0.949 & 0.419 & 0.804 \\
\hline Non-progressive & 36 & 26 & & & & \\
\hline Non-progressive & 50 & 12 & & & & \\
\hline \multicolumn{7}{|c|}{ Total refractive power } \\
\hline Progressive & 148 & 8 & 0.729 & 0.949 & 0.177 & 0.744 \\
\hline Non-progressive & 51 & 11 & & & & \\
\hline \multicolumn{7}{|l|}{ Pachymetry } \\
\hline Progressive & 138 & 18 & 0.720 & 0.885 & 0.306 & 0.762 \\
\hline Non-progressive & 43 & 19 & & & & \\
\hline
\end{tabular}

ophthalmologic fields, especially for the diagnosis, segmentation, classification, and staging of diseases. In recent years, there have been several recent studies on the prediction of disease progression, such as age-related macular degeneration (11), glaucoma $(12,13)$, diabetic retinopathy $(14,15)$, and other diseases $(16,17)$, using deep learning of the actual images. We firstly showed that deep learning was clinically beneficial for predicting the progression of the disease in keratoconic patients, (Kamiya K, Ayatsuka Y, Kato Y, et al. "Diagnostic Evaluation of Keratoconus Using Deep Learning (4th Report: Prediction of Disease Progression)", presented at the 73rd Annual Congress of Japan Clinical Ophthalmology, Kyoto, 2019, and Kamiya K. "Update on AI Application Using Corneal Tomography. Prediction of Keratoconus Progression Using Deep Learning." presented at the $35^{\text {th }}$ Congress of the Japanese Society of Cataract \& Refractive Surgery, Fukuoka, 2020). After that, it has been recently confirmed that the disease progression could be to some extent predicted by the use of the Scheimpflug camera maps (18), which was in good agreement with our current study. Chan et al. demonstrated that the swept-source As-OCT might be preferred over the Placido-Scheimpflug imaging owing to better repeatability of corneal measurements in keratoconic patients (19). Hence, we believe that the swept-source AsOCT is one of the best currently available technologies to precisely detect subtle progressive changes of the cornea in daily practice. To the best of our knowledge, this is the first study to evaluate the prediction accuracy of disease progression using deep learning of As-OCT images for keratoconus. These findings indicate that we can foresee the possibility of keratoconus progression, to some extent, with a single measurement. Based on the fact that there are no other definite means for identifying progressive keratoconus among keratoconic patients using corneal measurements during a single visit, even by corneal specialists, we believe that it will become an aid for decision-making of the surgical indication of the CXL treatment in daily practice.

Considering that the accuracy and the sensitivity were high (0.798 and 0.942 , respectively), but that the specificity was relatively low (0.419) in this study, we accept that it is still challenging to exactly predict keratoconus 

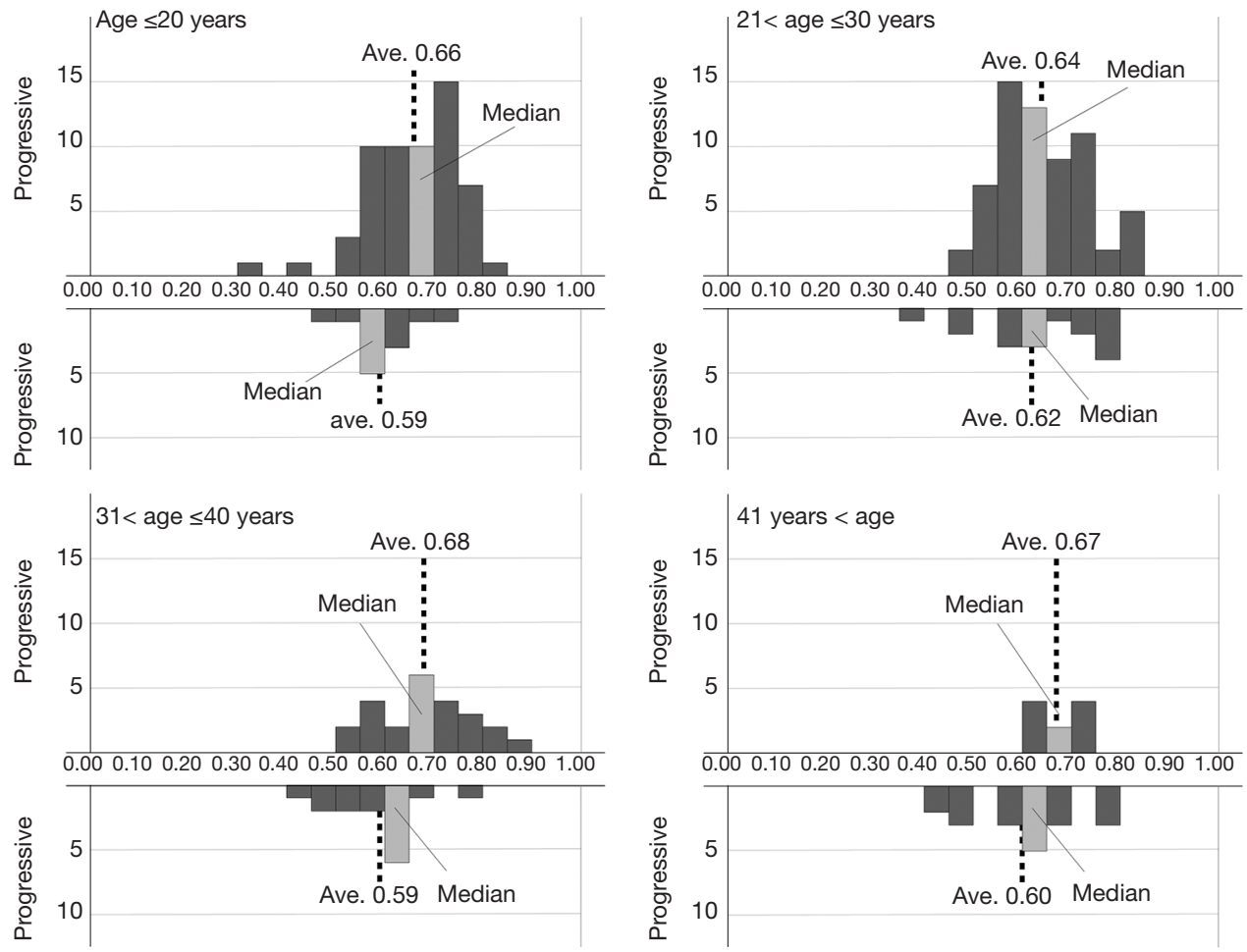

Figure 3 Histogram of anterior elevation classifier's output for progressive and non-progressive keratoconus, according to age subgroups.
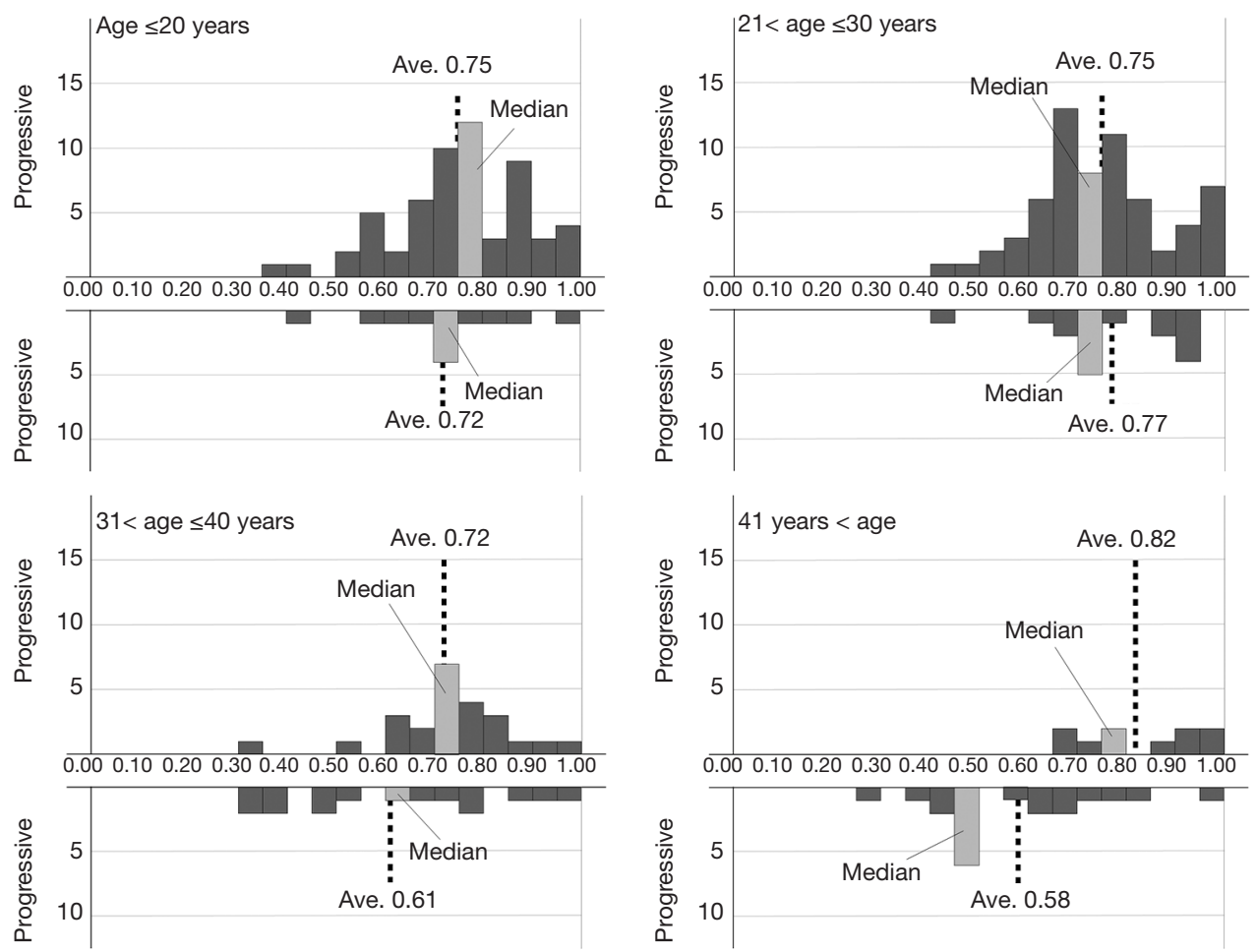

Figure 4 Histogram of anterior curvature classifier's output for progressive and non-progressive keratoconus, according to age subgroups. 

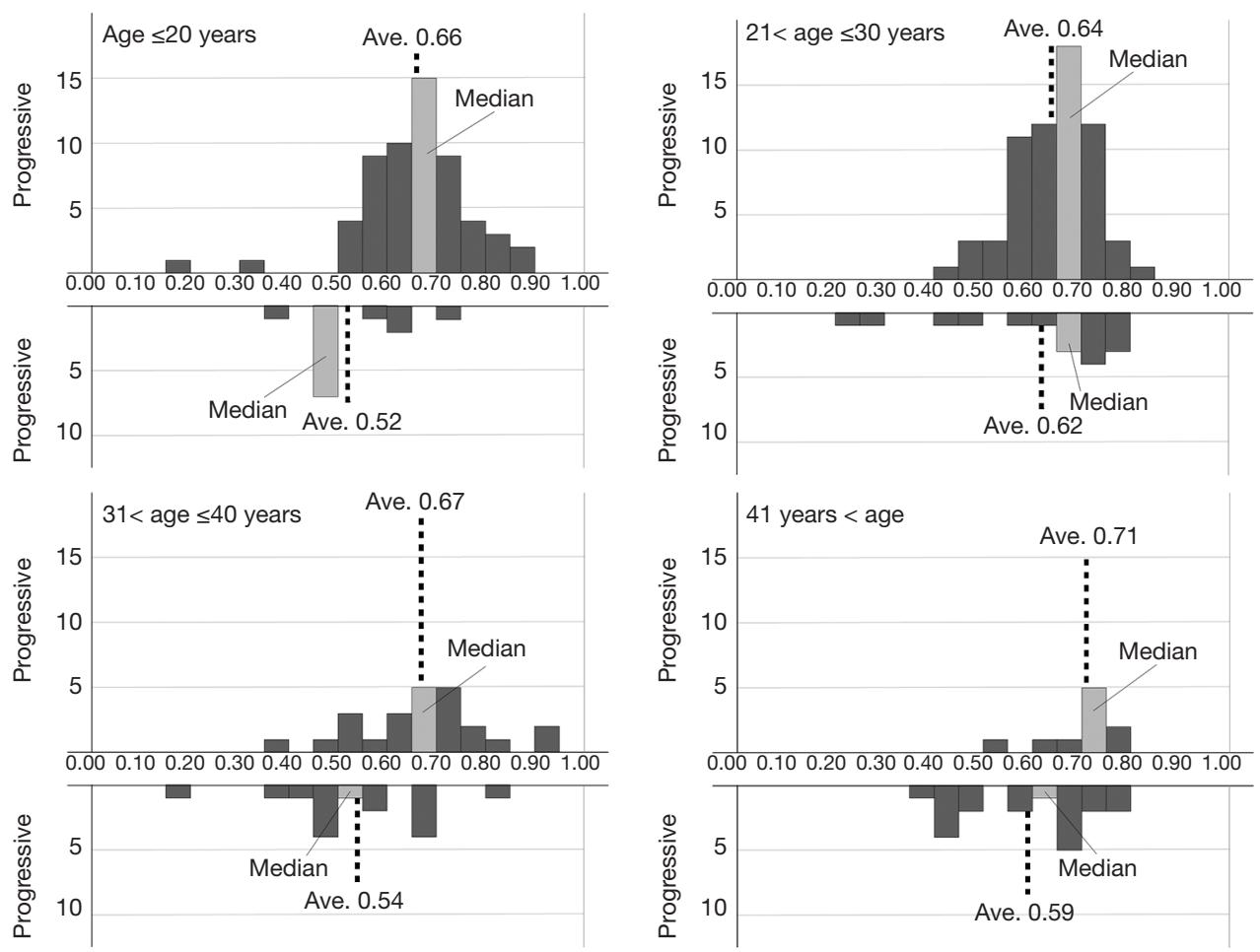

Figure 5 Histogram of posterior elevation classifier's output for progressive and non-progressive keratoconus, according to age subgroups.
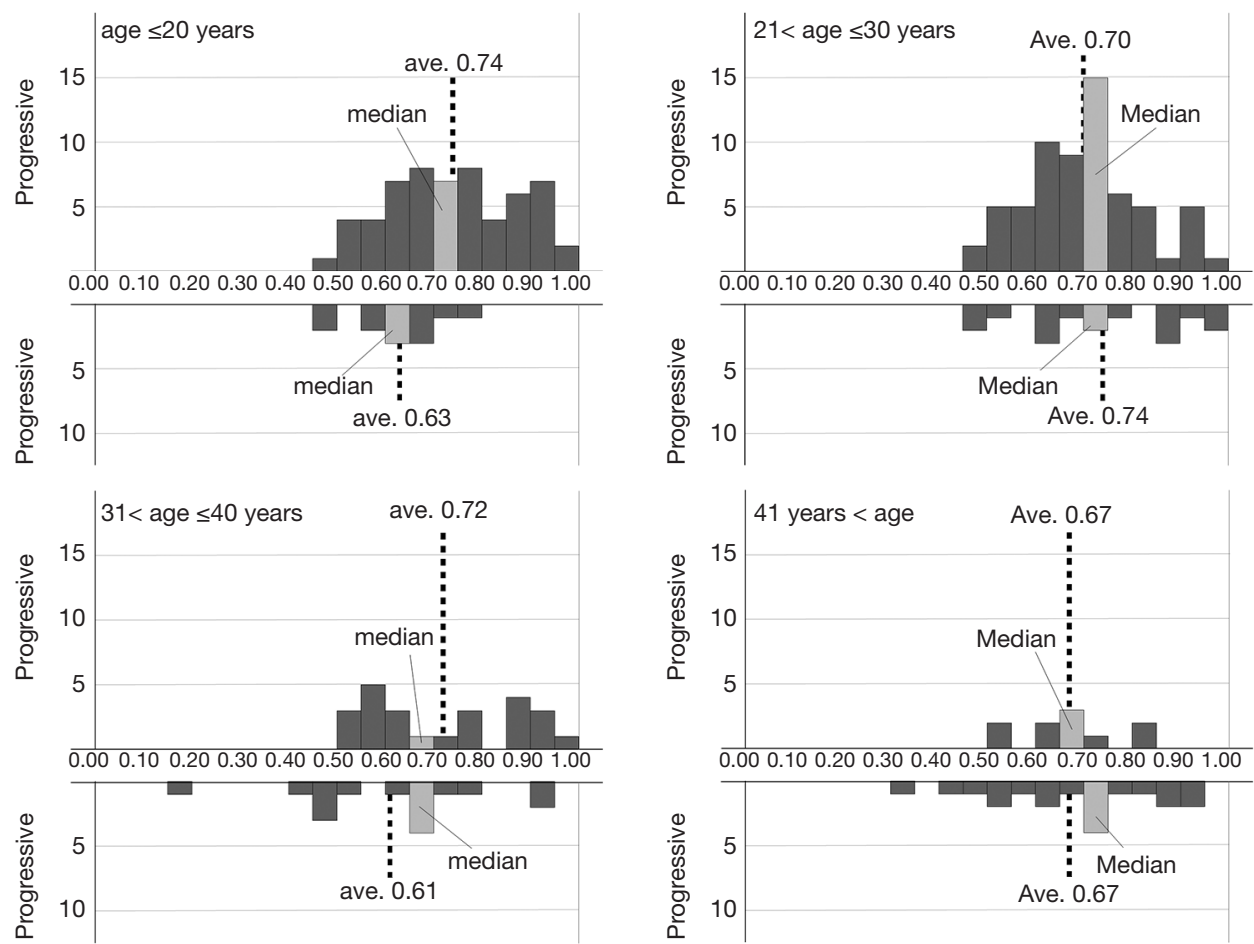

Figure 6 Histogram of posterior curvature classifier's output for progressive and non-progressive keratoconus, according to age subgroups. 

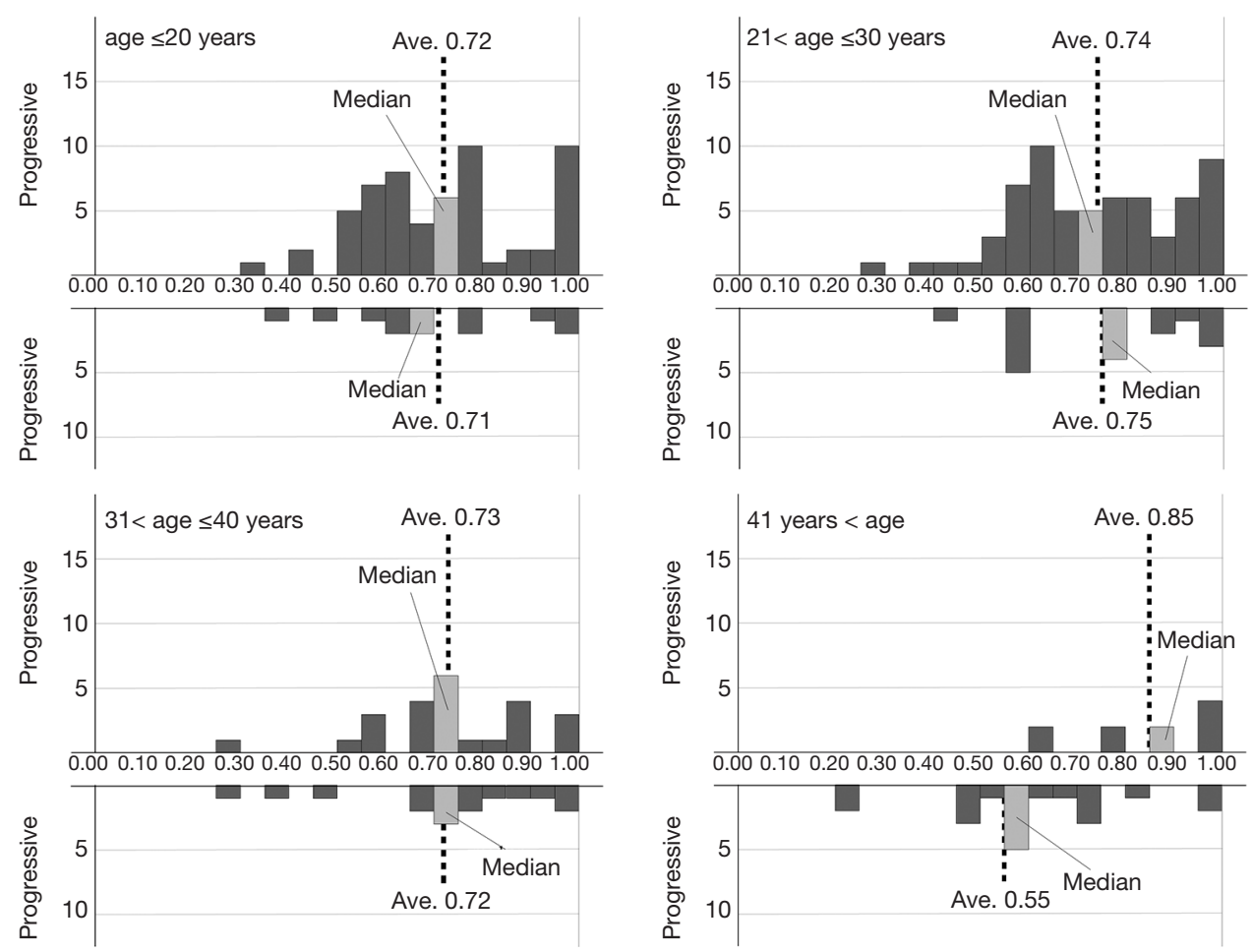

Figure 7 Histogram of total refractive power classifier's output for progressive and non-progressive keratoconus, according to age subgroups.
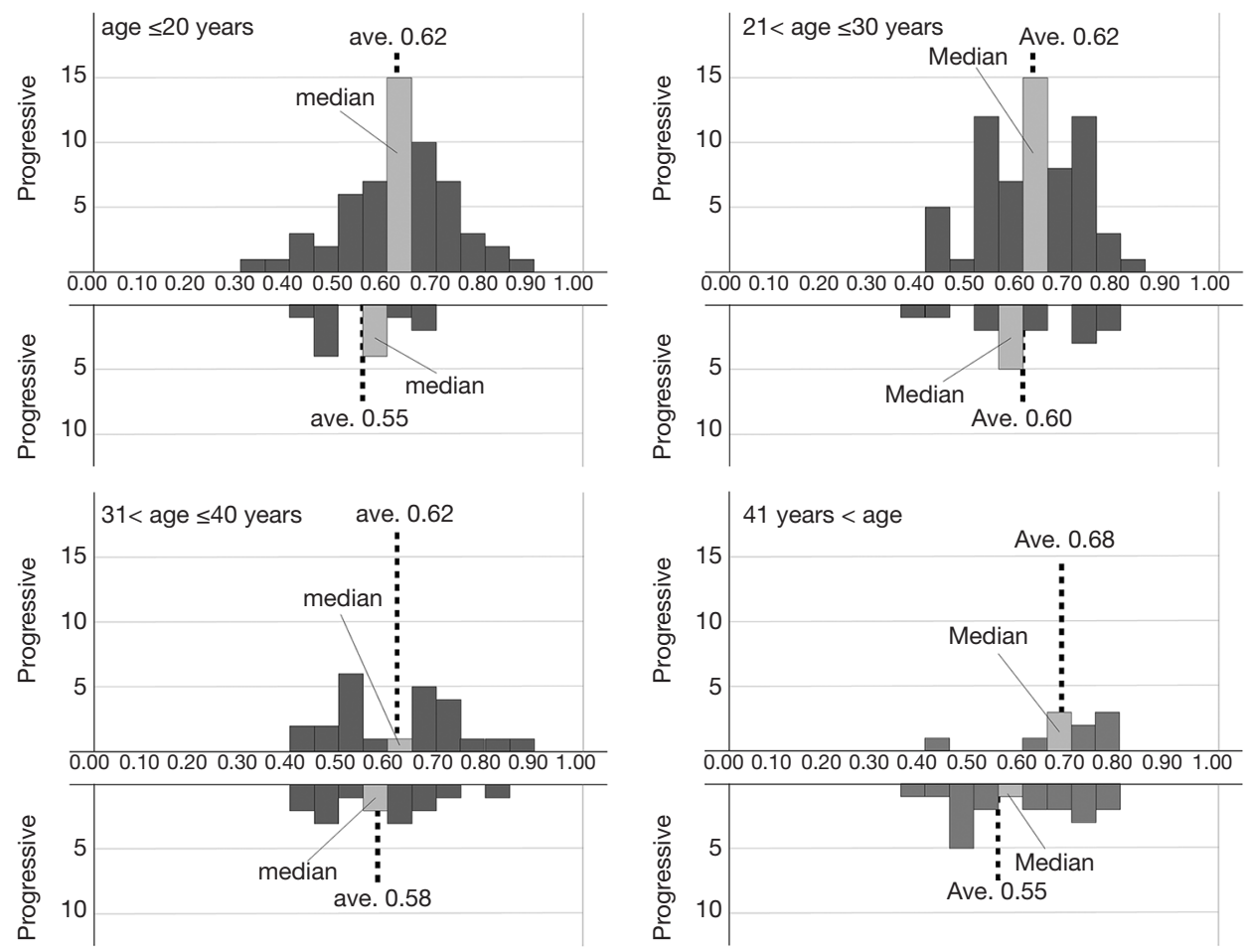

Figure 8 Histogram of pachymetry classifier's output for progressive and non-progressive keratoconus, according to age subgroups. 
progression. It has been shown that patient age plays a critical role in keratoconus progression in a clinical setting $(9,20)$. Therefore, we adjusted the deep learning algorithm based on age subgroups. As a consequence, the accuracy, sensitivity, and specificity of this prediction test have been much improved by adjustment of the learning algorithm based on patient age subgroups. Since there are so far no other established means to forecast deterioration during a single visit, we assume that the combined use of deep learning and As-OCT images with an algorithm adjusted based on age will hold a promise for improving the accuracy of keratoconus progression predictions.

This study is burdened with several limitations. Firstly, we did not perform any external validation studies with a different keratoconic population. However, we applied a $\mathrm{k}$-fold cross validation, one of the most prevalent validation techniques, to predict the progression of the disease in this pilot study, since this technique can not only improve the general applicability of the data but also handle the limited data efficiently, as published in many scientific journals. Secondly, we did not confirm the repeatability of the As-OCT measurements for keratoconus in this study. However, we recently reported that even keratoconic eyes showed high agreements (intraclass correlation coefficients; 0.980 to 0.992 ) in anterior and posterior keratometry (21). Thirdly, we did not completely exclude the effect of rigid or soft contact lenses on the corneal measurements with the As-OCT, although we asked the patients to stop wearing rigid gas permeable lenses at least for 3 weeks before this assessment. It is clinically impractical for these patients to stop wearing contact lenses for a long period of time, in consideration of their daily life activities. Fourthly, the sample size was relatively small, especially in nonprogressive keratoconus, since keratoconic patients tended to show progression during the long-term follow-up period, especially in the university hospital setting. Fifthly, it is still difficult to accurately diagnose keratoconus by ophthalmologists, and its diagnosis can be influenced not only by the definition but also by the severity of the disease. We still need to have ophthalmologists read the same dataset and compare the performance of this model with the ophthalmologists' readings.

In summary, our findings showed that deep learning of the As-OCT images was beneficial for discriminating the presence or absence of disease progression. Since it is still challenging for any ophthalmologist to predict keratoconus progression during a single visit, this tool could aid decision-making regarding CXL procedures. A further external validation study with another keratoconic population is still necessary to assure the authenticity of our results.

\section{Acknowledgments}

This study has been presented both at the 73rd Annual Congress of Japan Clinical Ophthalmology, Kyoto, 2019, and at the 35th Congress of the Japanese Society of Cataract \& Refractive Surgery, Fukuoka, 2020.

Funding: This work was in part supported by Grants-in-Aid for Scientific Research (Grant number 21K09706).

\section{Footnote}

Reporting Checklist: The authors have completed the STARD reporting checklist. Available at https://dx.doi. org/10.21037/atm-21-1772

Data Sharing Statement: Available at https://dx.doi. org/10.21037/atm-21-1772

Conflicts of Interest: All authors have completed the ICMJE uniform disclosure form (available at https://dx.doi. org/10.21037/atm-21-1772). KK reports grants from Alcon, grants from Johnson \& Johnson Vision, grants from Mitsui Chemical, consulting fees from Eye Lens Ltd., personal fees from STAAR Surgical, personal fees from Carl Zeiss Meditec AG, personal fees from Santen Pharmaceutical, personal fees from Senjyu Pharmaceutical, personal fees from AMO, personal fees from Kowa Company, personal fees from Otsuka Pharmaceutical, outside the submitted work. Nobuyuki Shoji reports grants, personal fees and non-financial support from TOMEY CORPORATION., non-financial support from RE Medical Inc., during the conduct of the study; grants and personal fees from KOWA PHARMACEUTICAL COMPANY LTD., grants, and personal fees from Otsuka Pharmaceutical Co., Ltd., grants and personal fees from Santen Pharmaceutical Co., Ltd., grants from CHUO SANGIO CO., grants from HOYA Corporation, grants from Alcon Japan Ltd., grants and personal fees from Alcon Pharmaceuticals, grants, and personal fees from SENJU PHARMACEUTICAL CO., LTD., grants from Ogura Inc., grants and personal fees from Pfizer Japan Inc., outside the submitted work. YM reports grants and personal fees from Alcon, grants from AMO, grants and personal fees from HOYA, grants, and personal fees from Senju Pharmaceutical, grants 
from Shionogi, grants and personal fees from Santen Pharmaceutical, grants, and personal fees from Novartis, grants from Wakamoto, grants from Kissei pharmaceutical, grants from Sucampo Pharma, personal fees from KOWA, outside the submitted work. Kazunori Miyata reports grants and personal fees from Alcon, grants and personal fees from AMO, grants and personal fees from HOYA, grants, and personal fees from Senju Pharmaceutical, grants from Shionogi, grants and personal fees from Santen Pharmaceutical, grants from Novartis, grants and personal fees from Wakamoto, grants from Kissei pharmaceutical, grants from Sucampo Pharma, personal fees from KOWA, personal fees from Ohtsuka pharmaceutical, personal fees from Chuosangio, personal fees from Tomey, personal fees from Staar, outside the submitted work. The other authors have no conflicts of interest to declare.

Ethical Statement: The authors are accountable for all aspects of the work in ensuring that questions related to the accuracy or integrity of any part of the work are appropriately investigated and resolved. This retrospective data review was approved by the Institutional Review Board of Miyata Eye Hospital (CS-315) and that of Tokyo University Hospital (2019354NI) and followed the tenets of the Declaration of Helsinki (as revised in 2013). Informed consent was obtained by the opt-out method.

Open Access Statement: This is an Open Access article distributed in accordance with the Creative Commons Attribution-NonCommercial-NoDerivs 4.0 International License (CC BY-NC-ND 4.0), which permits the noncommercial replication and distribution of the article with the strict proviso that no changes or edits are made and the original work is properly cited (including links to both the formal publication through the relevant DOI and the license). See: https://creativecommons.org/licenses/by-nc-nd/4.0/.

\section{References}

1. Godefrooij DA, de Wit GA, Uiterwaal CS, et al. Agespecific Incidence and Prevalence of Keratoconus: A Nationwide Registration Study. Am J Ophthalmol 2017;175:169-72.

2. Al-Amri AM. Prevalence of Keratoconus in a Refractive Surgery Population. J Ophthalmol 2018;2018:5983530.

3. Papali'i-Curtin AT, Cox R, Ma T, et al. Keratoconus Prevalence Among High School Students in New Zealand. Cornea 2019;38:1382-9.
4. Wollensak G, Spoerl E, Seiler T. Riboflavin/ultravioleta-induced collagen crosslinking for the treatment of keratoconus. Am J Ophthalmol 2003;135:620-7.

5. LeCun Y, Bottou L, Bengio Y, et al. Gradient-based learning applied to document recognition. Proc IEEE 1998;86:2278-324.

6. Samy El Gendy NM, Li Y, Zhang X, et al. Repeatability of pachymetric mapping using fourier domain optical coherence tomography in corneas with opacities. Cornea 2012;31:418-23.

7. Aptel F, Chiquet C, Gimbert A, et al. Anterior segment biometry using spectral-domain optical coherence tomography. J Refract Surg 2014;30:354-60.

8. Kamiya K, Ayatsuka Y, Kato Y, et al. Keratoconus detection using deep learning of colour-coded maps with anterior segment optical coherence tomography: a diagnostic accuracy study. BMJ Open 2019;9:e031313.

9. Rabinowitz YS. Keratoconus. Surv Ophthalmol 1998;42:297-319.

10. Chollet. Xception: Deep Learning with Depthwise Separable Convolutions. Honolulu, HI: 2017 IEEE Conference on Computer Vision and Pattern Recognition (CVPR), 2017:1800-7.

11. Russakoff DB, Lamin A, Oakley JD, et al. Deep Learning for Prediction of AMD Progression: A Pilot Study. Invest Ophthalmol Vis Sci 2019;60:712-22.

12. Wen JC, Lee CS, Keane PA, et al. Forecasting future Humphrey Visual Fields using deep learning. PLoS One 2019;14:e0214875.

13. Berchuck SI, Mukherjee S, Medeiros FA. Estimating Rates of Progression and Predicting Future Visual Fields in Glaucoma Using a Deep Variational Autoencoder. Sci Rep 2019;9:18113.

14. Arcadu F, Benmansour F, Maunz A, et al. Deep learning algorithm predicts diabetic retinopathy progression in individual patients. NPJ Digit Med 2019;2:92.

15. Hua CH, Huynh-The T, Kim K, et al. Bimodal learning via trilogy of skip-connection deep networks for diabetic retinopathy risk progression identification. Int J Med Inform 2019;132:103926.

16. Taylor S, Brown JM, Gupta K, et al. Monitoring Disease Progression With a Quantitative Severity Scale for Retinopathy of Prematurity Using Deep Learning. JAMA Ophthalmol 2019;137:1022-8.

17. Schmidt-Erfurth U, Bogunovic H, Grechenig C, et al. Role of Deep Learning-Quantified Hyperreflective Foci for the Prediction of Geographic Atrophy Progression. Am J Ophthalmol 2020;216:257-70. 
18. Kato N, Masumoto H, Tanabe M, et al. Predicting Keratoconus Progression and Need for Corneal Crosslinking Using Deep Learning. J Clin Med 2021;10:844.

19. Chan TCY, Biswas S, Yu M, et al. Comparison of corneal measurements in keratoconus using swept-source optical coherence tomography and combined Placido-Scheimpflug imaging. Acta Ophthalmol 2017;95:e486-94.

Cite this article as: Kamiya K, Ayatsuka Y, Kato Y, Shoji N, Miyai T, Ishii H, Mori Y, Miyata K. Prediction of keratoconus progression using deep learning of anterior segment optical coherence tomography maps. Ann Transl Med 2021;9(16):1287. doi: $10.21037 /$ atm-21-1772
20. Mas Tur V, MacGregor C, Jayaswal R, et al. A review of keratoconus: Diagnosis, pathophysiology, and genetics. Surv Ophthalmol 2017;62:770-83.

21. Saito A, Kamiya K, Fujimura F, et al. Comparison of reproducibility of corneal measurements by anterior segment optical coherence tomography in keratoconus and normal eyes. Japanese Journal of Visual Science 2020;14:80-5. 